\title{
Neutron Capture Cross Sections of ${ }^{194} \mathrm{Hg}$ and the Decays of ${ }^{195} \mathrm{Hg}$
}

\author{
S. F. Dorsett and K. S. Krane \\ Department of Physics, Oregon State University, Corvallis OR 97331 U.S.A.
}

The thermal cross section and resonance integral have been determined for radiative neutron capture by radioactive ${ }^{194} \mathrm{Hg}$ by observing the $\gamma$ rays emitted in the decay of ${ }^{195} \mathrm{Hg}$. Captures leading to the low-spin isomer of ${ }^{195} \mathrm{Hg}$ give $\sigma=877 \mathrm{~b}$ and $I=10,270 \mathrm{~b}$, while captures leading to the high-spin isomer give $\sigma=49 \mathrm{~b}$ and $I=608 \mathrm{~b}$. Energies and intensities of the $\gamma$ rays emitted in the decays of ${ }^{195} \mathrm{Hg}$ produced by neutron capture have been obtained with increased precision relative to previous studies with sources produced by other reactions, leading to correspondingly improved values for $\beta$-decay branching intensities and energy levels in ${ }^{195} \mathrm{Au}$. New values for the half-lives of ${ }^{195 \mathrm{~g}} \mathrm{Hg}$ and ${ }^{195 \mathrm{~m}} \mathrm{Hg}$ are also reported.

Keywords: $\quad{ }^{194} \mathrm{Hg}$ neutron capture cross sections

${ }^{195 \mathrm{~g}} \mathrm{Hg},{ }^{195 \mathrm{~m}} \mathrm{Hg} \gamma$ ray spectroscopy

${ }^{195}$ Au energy levels 


\section{Introduction}

Studying neutron capture by radioactive nuclei presents considerable challenges compared with captures by stable nuclei. A radioactive sample of activity $1 \mu \mathrm{Ci}$ might contain perhaps $10^{12}-10^{14}$ nuclei, while even a milligram of a stable target contains $10^{20}$ nuclei. The activity produced in the capture by radioactive nuclei is thus in general much weaker than that produced in capture by stable nuclei, so that the experimenter is faced with searching for a weak activity in the presence of the original, much stronger activity. For this effort to succeed, it is necessary that the original activity be long-lived (thereby increasing the number of capturing nuclei per unit of activity) and that the activity produced in the capture be short-lived (thereby compensating for the relatively smaller number of capturing nuclei). Radioactive ${ }^{194} \mathrm{Hg}$ satisfies these criteria - the ratio of the ${ }^{194} \mathrm{Hg}$ and ${ }^{195} \mathrm{Hg}$ half-lives is about $3.7 \times 10^{5}$, which compensates for the relatively small number of captures produced. Moreover, there are both low-spin and high-spin activities produced in the capture, which allows a study of the differences in the capture cross sections leading to the two states. The present report describes measurements of the ${ }^{194} \mathrm{Hg}$ radiative neutron capture cross sections.

In the process of the cross-section measurements, spectroscopic data were obtained on the $\gamma$ rays emitted in the ${ }^{195} \mathrm{Hg}$ decays which surpassed the precision of the previously accepted values of the energies and intensities from these decays. This report includes the results of our spectroscopic studies, which allows the deduction of $\beta$-decay branching intensities and of energies of the excited states populated in the decays. As in the 
previously reported studies of the ${ }^{195} \mathrm{Hg}$ decays, which are summarized in the tabulations of the Nuclear Data Sheets (NDS) by Zhou (1999), the present samples contained a mixture of ${ }^{195 g} \mathrm{Hg}$ and ${ }^{195 \mathrm{~m}} \mathrm{Hg}$. However, neutron capture produces relatively less ${ }^{195 \mathrm{~m}} \mathrm{Hg}$ than the production reactions employed in the previous studies. The present spectroscopic data thus serve to complement the previous studies, particularly in separating the intensities of transitions produced in both the ground-state and isomeric decays.

Figure 1 shows a schematic view of the neutron capture process for ${ }^{194} \mathrm{Hg}$. The capture proceeds through high-lying resonances (mostly $1 / 2^{+}$, corresponding to s-wave capture). Following the emission of many (unobserved) primary and secondary $\gamma$ rays, the capture process leads to the formation of the $13 / 2^{+}$metastable state and the $1 / 2^{-}$ground state in ${ }^{195} \mathrm{Hg}$. The metastable state decays to the ground state with a $54.2 \%$ branch through $\gamma$ emission and electron conversion, and it $\beta$ decays to states in ${ }^{195} \mathrm{Au}$ with a $45.8 \%$ branch. 


\section{Experimental details}

The ${ }^{194} \mathrm{Hg}$ activity was produced at the Los Alamos Meson Physics Facility by $800-\mathrm{MeV}$ proton spallation reactions on a $\mathrm{Pb}$ target and was obtained in the form of $\mathrm{HNO}_{3}$ solution after the $\mathrm{Hg}$ activity was separated by ion exchange. The proton irradiation was done in the early 1980s and the separation in 1996, so there should be no activities present in the sample with half-lives less than a few years. The specific activity of the ${ }^{194} \mathrm{Hg}$ was approximately $1 \mu \mathrm{Ci}$ per $\mathrm{mL}$. Samples for neutron irradiation were prepared either in the liquid form or dried on aluminum foil and typically amounted to $0.1-0.2 \mu \mathrm{Ci}$ of the ${ }^{194} \mathrm{Hg}$ activity.

The samples were irradiated in the Oregon State University TRIGA reactor. Several different irradiation sites in the reactor were used: central core (nominal thermal and epithermal fluxes of respectively $1.0 \times 10^{13}$ and $3.0 \times 10^{11}$ neutrons $\mathrm{cm}^{-2} \mathrm{~s}^{-1}$ ), Cd-lined central core $\left(0\right.$ and $1.2 \times 10^{12}$ neutrons $\left.\mathrm{cm}^{-2} \mathrm{~s}^{-1}\right)$, thermal column $\left(6.4 \times 10^{10}\right.$ and $1.8 \times$ $10^{8}$ neutrons $\mathrm{cm}^{-2} \mathrm{~s}^{-1}$ ), and fast pneumatic transfer system, located on the outer rim of the core $\left(1.0 \times 10^{13}\right.$ and $3.5 \times 10^{11}$ neutrons $\left.\mathrm{cm}^{-2} \mathrm{~s}^{-1}\right)$. In the irradiations in the latter site, some samples were enclosed in a $\mathrm{Cd}$ box $(1.0 \mathrm{~mm}$ wall thickness) to isolate the epithermal neutrons. Typical irradiations lasted for 10 minutes in the pneumatic transfer system and from one to several hours in the other sites. All irradiations were accompanied by flux monitors. Primary flux monitors were $\mathrm{Au}$ and $\mathrm{Co}$ as dilute alloys (respectively $0.134 \%$ and $0.438 \%$ ) in thin $\mathrm{Al}$ metal foils; $\mathrm{Zr}$ metal foils served as secondary flux monitors. 
The $\gamma$ rays from the irradiated samples were observed with high-resolution Ge detectors (efficiency 35-40\% compared with $\mathrm{NaI}$ at $1332 \mathrm{keV}$, resolution $1.68 \mathrm{keV}$ at $1332 \mathrm{keV}$ ). The signals were analyzed with a digital spectroscopy system coupled to a desktop computer. Areas for the well isolated peaks used for the cross-section measurements were determined using the Gammavision software.

Efficiency calibration of the detectors was done using standardized sources of ${ }^{133} \mathrm{Ba}$ and ${ }^{152} \mathrm{Eu}$. Below $200 \mathrm{keV}$ the efficiency calibration was also characterized using reactorproduced sources of ${ }^{160} \mathrm{~Tb},{ }^{169} \mathrm{Yb}$, and ${ }^{182} \mathrm{Ta}$. The log-log dependence of efficiency on energy was fit with a $4^{\text {th }}$ degree polynomial below $250 \mathrm{keV}$ and with two different linear relationships above $250 \mathrm{keV}$ (one for the region $250-450 \mathrm{keV}$ and another for energies above $450 \mathrm{kev})$.

For the ${ }^{195} \mathrm{Hg}$ spectroscopy studies, a sample of $0.45 \mu \mathrm{Ci}$ of ${ }^{194} \mathrm{Hg}$ was irradiated to produce $6.6 \mu \mathrm{Ci}$ of ${ }^{195 \mathrm{~g}} \mathrm{Hg}$ and $0.19 \mu \mathrm{Ci}$ of ${ }^{195 \mathrm{~m}} \mathrm{Hg}$. To study the ${ }^{195 \mathrm{~g}} \mathrm{Hg} \gamma$ rays, the sample was counted for $20 \mathrm{~h}$ at a source-to-detector distance of $15 \mathrm{~cm}$ and then for another $20 \mathrm{~h}$ at $10 \mathrm{~cm}$. Another 4 days of counting at $10 \mathrm{~cm}$ allowed the accumulation of data on the ${ }^{195 \mathrm{~m}} \mathrm{Hg}$ decays. The spectra were analyzed using the peak-fitting code SAMPO (Aarnio, 1988) to determine energies and intensities.

The determination of the cross sections requires knowledge of the absolute decay intensities of the prominent $\gamma$ rays, which have been obtained from the spectroscopic 
studies discussed later in this paper. With energies given in $\mathrm{keV}$, the branching intensities are:

$$
\begin{aligned}
&{ }^{195 m} \mathrm{Hg}: 261.8(30.3 \%), 388.0(2.24 \%), 560.3(7.44 \%) \\
&{ }^{195 g} \mathrm{Hg}: 180.1(1.58 \%), 207.1(1.53 \%), 261.8(1.56 \%), 585.2(2.00 \%), \\
& 599.7(1.79 \%), 779.8(6.31 \%)
\end{aligned}
$$

The relative uncertainties in these values are substantial: $\pm 9.1 \%$ for the ${ }^{195 \mathrm{~g}} \mathrm{Hg}$ decay and $\pm 4.4 \%$ for the ${ }^{195 \mathrm{~m}} \mathrm{Hg}$ decay; as discussed in Section 4 below, these uncertainties originate with the uncertainties in the ground-state $\beta$ intensity $\left({ }^{195 \mathrm{~g}} \mathrm{Hg}\right.$ decay) and the isomeric transition intensity $\left({ }^{195 \mathrm{~m}} \mathrm{Hg}\right.$ decay $)$, which affect the overall normalization of the intensities. Within these uncertainties, the absolute $\gamma$ ray intensities agree with the NDS values.

In the cross section measurements, the determination of the ${ }^{194} \mathrm{Hg}$ activity (and thus the number of capturing ${ }^{194} \mathrm{Hg}$ nuclei) used the strong $\gamma$ rays at $293.5 \mathrm{keV}(10.6 \%)$ and 328.5 $\mathrm{keV}(61.4 \%)$. The relative uncertainty in each of these intensities is about $1 \%$ (Dorsett and Krane, to be published). 


\section{Cross section results}

For each irradiation, the amounts of the ${ }^{195 g} \mathrm{Hg}$ and ${ }^{195 \mathrm{~m}} \mathrm{Hg}$ activities were determined from the $\gamma$-ray intensities using the peaks listed in Section 2. A half-life correction was applied to compensate for decay from the end of bombardment to the time of counting (which ranged from 30 minutes for the samples produced in the pneumatic transfer system to overnight for the samples irradiated in the core). The end-of-bombardment activity $a$ depends on the cross sections according to

$$
a=N\left(\sigma \phi_{\mathrm{th}}+I \phi_{\mathrm{epi}}\right)\left(1-e^{-\lambda \mathrm{t}_{\mathrm{i}}}\right)
$$

where $N$ is the number of ${ }^{194} \mathrm{Hg}$ target nuclei in the irradiated sample, $\phi_{\mathrm{th}}$ and $\phi_{\mathrm{epi}}$ are the thermal and epithermal neutron fluxes, and $t_{\mathrm{i}}$ is the irradiation time.

In Eq. 1, $\sigma$ represents the effective thermal cross section (that is, the $2200-\mathrm{m} / \mathrm{s}$ value). The resonance structure of ${ }^{194} \mathrm{Hg}$ has not been studied, so it is not known whether there are any broad or low-lying neutron resonances that might cause the cross section in the thermal region to deviate from $1 / v$ behavior, that is, whether the Westcott $g$-factor differs from unity (Mughabghab, 2006). Such cases are rare, and none of the nuclei in the vicinity of ${ }^{194} \mathrm{Hg}$ show this behavior. In the absence of contrary information, we have assumed that the $1 / v$ behavior is followed for ${ }^{194} \mathrm{Hg}$. 
The effective resonance integral $I$ includes a small contribution from the $1 / v$ region. Assuming the Cd cut-off energy to be about $0.5 \mathrm{eV}$, this contribution amounts to about $0.45 \sigma$; the corrected resonance integral $I^{\prime}$ is then (Mughabghab, 2006)

$$
I^{\prime}=I-0.45 \sigma
$$

Because the resonance integral is usually larger than the thermal cross section (about an order of magnitude larger for most nuclei), this correction is small and in almost all cases within about one standard deviation of the values of the resonance integrals. However, it is important to make this correction for the $\mathrm{Au}$ and Co flux monitors to avoid introducing additional systematic uncertainties in the flux determinations. For our flux monitors we have assumed the following cross sections: $\sigma(\mathrm{Au})=98.65 \pm 0.09 \mathrm{~b}, I(\mathrm{Au})=1550 \pm 28 \mathrm{~b}$, $\sigma(\mathrm{Co})=37.18 \pm 0.06 \mathrm{~b}, I(\mathrm{Co})=74 \pm 2 \mathrm{~b}($ Mughabghab, 2006). Use of these flux monitors introduces a negligible uncertainty in the thermal flux but a 2-3\% uncertainty in the epithermal flux, which propagates into a similar uncertainty in the deduced resonance integrals.

Uncertainties in the deduced cross sections depend on a number of measurement-related factors, including the flux determinations, detector efficiency calibrations, counting statistics, and relative branching ratios. The influence of some of these factors (for example, the flux determinations and the detector efficiency calibrations) is reduced because the measurement is in effect the determination of the ratio between the activity measured with the ${ }^{194} \mathrm{Hg}$ sample and that measured with the flux monitors. Overall these factors combine to give a minimum uncertainty in the range of $4-5 \%$ in the thermal cross 
sections. The absolute branching ratio normalization of the ${ }^{195} \mathrm{Hg} \gamma$ rays introduces a possible systematic uncertainty of $9.1 \%$ for ${ }^{195 g} \mathrm{Hg}$ and $4.4 \%$ for ${ }^{195 \mathrm{~m}} \mathrm{Hg}$.

The determination of the number of irradiated nuclei from the ${ }^{194} \mathrm{Hg}$ activity requires the value of the half-life of ${ }^{194} \mathrm{Hg}$. There are 2 measured values reported: $367 \pm 55$ y (Probst et al., 1979) and $520 \pm 32$ y (Hornshoj et al., 1981). The former measurement used excitation functions from proton-induced reactions on Au to determine the yield of ${ }^{194} \mathrm{Hg}$, while the second measurement used the specific activity method with an isotope separator to collect and count the number of ${ }^{194} \mathrm{Hg}$ nuclei. The NDS evaluation regarded these as incommensurate values and recommended a value of $444 \pm 77 \mathrm{y}$ to span the range of the measured values. However, both values need to be corrected owing to their use of branching ratios for the $328.5 \mathrm{keV} \gamma$ ray (respectively, $63 \%$ and $67 \%$ ) that differ from the current value (61.4\%). Making the corrections, the measured half-lives become, respectively, $358 \pm 55 \mathrm{y}$ and $477 \pm 32 \mathrm{y}$. The uncertainty in the latter value includes a statistical contribution ( $\pm 20 \mathrm{y})$ and a systematic contribution ( $\pm 25 \mathrm{y})$, with the latter in part including uncertainties in the decay scheme parameters. With the current value of the $328.5 \mathrm{keV}$ branching ratio known to $\pm 1 \%$, it is clear that these systematic uncertainties are perhaps somewhat overestimated. The recalculated values are now less incommensurate and differ by slightly more than one standard deviation, with a weighted average of $447 \pm 28 \mathrm{y}$. The value of Hornshoj et al. dominates in the calculation of the average value, owing to its smaller uncertainty reflecting perhaps a more direct technique less subject to systematic uncertainties. In our determination of the amount of ${ }^{194} \mathrm{Hg}$ present in our samples, we have adopted this average value with its uncertainty of \pm 
6.3\%. In order to permit future improvements in the half-life measurement to produce more precise values for the cross sections from the present data, the uncertainty in the deduced cross sections has been separated into 2 components: one due to the ${ }^{194} \mathrm{Hg}$ halflife and another due to all other factors, the latter being dominated by the uncertainties in the ${ }^{195} \mathrm{Hg}$ branching ratios.

The values quoted for the ground-state cross sections represent processes leading directly to the ground state with the contributions of the isomeric state subtracted out. This contribution is very small, partly because of the smaller cross section of the isomeric state and partly due to its longer half-life.

Our final values for the cross sections, representing the average of several measurements using the different reactor irradiation sites, are:

$$
\begin{aligned}
& \sigma\left({ }^{194} \mathrm{Hg} \rightarrow{ }^{195 \mathrm{~g}} \mathrm{Hg}\right)=877 \pm 75 \pm 55 \mathrm{~b} \\
& I\left({ }^{194} \mathrm{Hg} \rightarrow{ }^{195 \mathrm{~g}} \mathrm{Hg}\right)=10,270 \pm 880 \pm 640 \mathrm{~b} \\
& \sigma\left({ }^{194} \mathrm{Hg} \rightarrow{ }^{195 \mathrm{~m}} \mathrm{Hg}\right)=49 \pm 3 \pm 3 \mathrm{~b} \\
& I\left({ }^{194} \mathrm{Hg} \rightarrow{ }^{195 \mathrm{~m}} \mathrm{Hg}\right)=608 \pm 35 \pm 38 \mathrm{~b}
\end{aligned}
$$

The first uncertainty represents the net effect (combined in quadrature) of the uncertainties in the branching ratios, detector efficiencies, flux determinations, and (in the case of the thermal cross sections) the uncertainty in the resonance integral. The second uncertainty represents the effect of the uncertainty in the ${ }^{194} \mathrm{Hg}$ half-life. 
${ }^{194} \mathrm{Au}$ is in secular equilibrium with ${ }^{194} \mathrm{Hg}$ in our samples. Given the relative half-lives, the number of ${ }^{194} \mathrm{Au}$ atoms present is of the order of $10^{-5}$ times the number of ${ }^{194} \mathrm{Hg}$ atoms. Accordingly, it is not possible in our experiments to observe neutron capture by ${ }^{194} \mathrm{Au}$ to produce $186-\mathrm{d}{ }^{195} \mathrm{Au}$. Indeed, in our experiments the ${ }^{195} \mathrm{Au}$ activity increases with time (as evidenced by the counting rate of the $98.9-\mathrm{keV} \gamma$ ray emitted in the decay) consistent with its entire production resulting from the decay of ${ }^{195} \mathrm{Hg}$. 


\section{Spectroscopy of the ${ }^{195 g} \mathrm{Hg}$ and ${ }^{195 \mathrm{~m}} \mathrm{Hg}$ decays}

Figures 2 and 3 show a sample $\gamma$-ray spectrum from an irradiated sample. In addition to the lines from the decays of ${ }^{195 \mathrm{~g}, \mathrm{~m}} \mathrm{Hg}$, the spectra show lines from the original ${ }^{194} \mathrm{Hg}$ source material along with ${ }^{197 \mathrm{~g}, \mathrm{~m}} \mathrm{Hg}$. The ${ }^{197} \mathrm{Hg}$ arises from neutron capture by stable ${ }^{196} \mathrm{Hg}$, which was introduced into the sample material through the $\mathrm{Hg}$ carrier employed in the separation process. Despite its small abundance $(0.15 \%)$, the large capture cross sections of ${ }^{196} \mathrm{Hg}$ result in observable intensities of the ${ }^{197} \mathrm{Hg} \gamma$ ray lines.

Our deduced energies and intensities are listed in Tables 1 and 2 along with the compiled data from the Nuclear Data Sheets (Zhou, 1999). Energy calibrations were done by counting the ${ }^{195} \mathrm{Hg}$ samples simultaneously with ${ }^{133} \mathrm{Ba}$ and ${ }^{152} \mathrm{Eu}$ calibration sources. Energies of the calibration lines were taken from values recommended by Helmer and van der Leun (2000) and by the BIPM (2006). The combination of the energy uncertainties of the stronger calibration lines (often a few eV) and the stronger $\mathrm{Hg}$ lines would often lead to net energy uncertainties smaller than $10 \mathrm{eV}$. We feel that this underestimates the total energy uncertainty, so we have set the minimum energy uncertainty at $10 \mathrm{eV}$ for $\mathrm{Hg}$ peaks whose energies could be precisely determined by direct comparison with the $\mathrm{Ba}$ and Eu calibration lines and at $14 \mathrm{eV}$ for peaks whose energies were determined by comparison with secondary standards $\left({ }^{194} \mathrm{Au}\right.$ or the stronger ${ }^{195} \mathrm{Hg}$ lines). 
The NDS evaluation represents a consensus of the work of Frána et al. (1971), who used a source prepared by the ${ }^{3} \mathrm{He}, 2 \mathrm{n}$ reaction on ${ }^{194} \mathrm{Pt}$; Vieu et al. (1973), who produced their source by proton spallation of $\mathrm{Au}$; and Farzine and Buttlar (1974), whose source was produced by bremsstrahlung on ${ }^{196} \mathrm{Hg}$. The ratio of ${ }^{195 m} \mathrm{Hg}$ to ${ }^{195 \mathrm{~g}} \mathrm{Hg}$ activity in these three works was respectively $1.3,1.3$, and 0.16 , compared with 0.03 in the present work. Thus, while the present work suffers from an inability to observe the very weak lines in the ${ }^{195 \mathrm{~m}} \mathrm{Hg}$ decay, we are able to obtain more precise separation of the ${ }^{195 \mathrm{~g}} \mathrm{Hg}$ lines from those produced in the ${ }^{195 \mathrm{~m}} \mathrm{Hg}$ decay.

All lines listed in Table 1 with intensities above 0.2 showed decays consistent with the half-life of ${ }^{195 \mathrm{~g}} \mathrm{Hg}$ except those at 811.5 and $1049.3 \mathrm{keV}$, which include unresolvable contributions from lines in the ${ }^{194} \mathrm{Au}$ decay. Table 2 includes only results for the lines in the ${ }^{195 \mathrm{~m}} \mathrm{Hg}$ decay with intensities of about $1 \%$ and above (about $1 / 3$ of the transitions listed in the NDS evaluation). Because of the small amount of ${ }^{195 \mathrm{~m}} \mathrm{Hg}$ activity produced in neutron capture, we were not able to observe the many weak lines in this decay. Except for $207.1 \mathrm{keV}$, which contains a significant component from the ${ }^{195 \mathrm{~g}} \mathrm{Hg}$ decay, and $467.4 \mathrm{keV}$, which overlaps a line from a ${ }^{192}$ Ir impurity, all lines listed in Table 2 with intensities greater than $2 \%$ appear to follow the expected 41.6 -h half-life of ${ }^{195 m} \mathrm{Hg}$.

Overall the agreement with previous work is good, with uncertainties in our data considerably smaller than those of previous work. Owing mostly to the presence of ${ }^{194} \mathrm{Hg}$ in our sample, several of the very weak lines in the ${ }^{195 g} \mathrm{Hg}$ decay could not be isolated. 
The doublet at $841 \mathrm{keV}$ has an expected separation of only $0.1 \mathrm{keV}$, which is too small to be resolved in our fits to that peak.

The line at $401.7 \mathrm{keV}$ is an unresolved doublet with components from both ${ }^{195 \mathrm{~g}} \mathrm{Hg}$ and ${ }^{195 \mathrm{~m}} \mathrm{Hg}$ separated in energy by less than $0.1 \mathrm{keV}$. However, because of the small activity of ${ }^{195 \mathrm{~m}} \mathrm{Hg}$ relative to ${ }^{195 \mathrm{~g}} \mathrm{Hg}$ in our sample compared with those of previous works, the ${ }^{195 \mathrm{~m}} \mathrm{Hg}$ contribution to this line is negligible (less than 2\%). As shown in Fig. 4, the ratio of the intensity of the $401.7-\mathrm{keV}$ line to that of the $779.8-\mathrm{keV}$ line remained constant over more than 3 half-lives. We are therefore able to assign essentially all of the observed intensity of this line to ${ }^{195 \mathrm{~g}} \mathrm{Hg}$. By subtracting this contribution from the reported $\mathrm{g}+\mathrm{m}$ intensity from previous works, we deduce the intensity of the 401.7-keV line in the ${ }^{195 \mathrm{~m}} \mathrm{Hg}$ decay to be $0.07 \pm 0.05$ (relative to 100 units for the intensity of the $560.3-\mathrm{keV}$ line) from the data of Frána et al. and $0.06 \pm 0.03$ from Vieu et al.

In addition to the 401.7-keV line, the present work differs from previous work in the intensity of the $869.0-\mathrm{keV}$ line in the ${ }^{195 \mathrm{~g}} \mathrm{Hg}$ decay. The NDS value of $0.051 \pm 0.019$ shown in Table 4 is from Farzine and Buttlar; other values are $0.245 \pm 0.041$ from Vieu et al. and $0.045 \pm 0.020$ from Frána et al. Our value for this relative intensity has been corrected for a small contribution from a very weak ${ }^{194} \mathrm{Hg}$ line (the correction amounting to $5 \%$ at the start of counting). The remaining intensity shows a constant relationship to the 779.8-keV intensity over three decay half-lives, as shown in Fig. 4, suggesting that it is probably associated with the decay of ${ }^{195 \mathrm{~g}} \mathrm{Hg}$. No line at that energy appears in the ${ }^{195 m} \mathrm{Hg}$ decay, nor are there any known impurity activities that would produce this line. 
The disagreement among the various studies in accounting for the intensity of this line is therefore puzzling. A further puzzlement, discussed below, is the disagreement between the measured energy of this line and the energy difference of the levels corresponding to its suggested placement in the decay scheme.

The energy levels in ${ }^{195} \mathrm{Au}$ and the $\beta$ intensities deduced from our energies and intensities from the ${ }^{195 g} \mathrm{Hg}$ decay are listed in Table 3. The $\beta$ intensities were calculated based on the difference between the net gamma plus internal conversion intensity populating and depopulating each level, using the internal conversion coefficients tabulated in the NDS evaluation. In cases in which first-forbidden decays occur to final states with uncertain spin assignments, the $\log f t$ values were calculated assuming nonunique decays. For the $318.5 \mathrm{keV}$ level, we have used the NDS total intensity $(30.7 \pm 3.5)$ of the $56.8 \mathrm{keV}$ transition to obtain the total intensity depopulating the level, the range of which gives the upper limit on any possible $\beta$ feeding for that level. To normalize the $\beta$ intensities, we have assumed the total $\gamma$ plus conversion electron intensity feeding the ground state to be $88 \pm 8 \%$ as given in the NDS evaluation. This value is obtained from the ground-state to ground-state $\beta$ intensity of $12 \pm 8 \%$ deduced in the NDS evaluation based on systematics of $\beta$ decays in neighboring $\mathrm{Hg}$ isotopes $(A=193,197,199)$. We note that the NDS computes the $\beta$ intensities also under a slightly different assumption, namely that the $\beta$ feeding to the ground state is $10 \pm 2 \%$. There is very little difference in the magnitudes of the $\beta$ intensities calculated under the two different assumptions, but there is a considerable difference in their uncertainties. We have calculated the $\beta$ intensities using the larger of the uncertainties. The two largest contributions to the uncertainties in the $\beta$ 
intensities are the ground-state $\beta$ feeding and the uncertainty in the intensity of the 61.4$\mathrm{keV} \gamma$ ray which (because of its large conversion coefficient) has a significant effect on the overall normalization of the $\beta$ intensities.

The previous $\gamma$-ray placements agree with the more precise level energies of the present data set with two exceptions: (1) The transition at $868.987 \mathrm{keV}$ disagrees with its previously suggested placement between levels $\mathrm{K}$ and $\mathrm{C}(\Delta E=869.228 \mathrm{keV})$ by more than 4 standard deviations. (The energy of level $\mathrm{K}$ was computed based only on the 1049.3- and 671.2-keV transitions, which are in very good agreement with one another.) The disagreements of the various studies concerning the intensity of the $869.0-\mathrm{keV}$ transition as discussed above, along with the discordant energies, perhaps suggest an additional component in the $869.0-\mathrm{keV}$ peak, but that peak is too weak in the present studies to reveal any structure. (2) The energies of level O computed from the 1091.5and $1353.7-\mathrm{keV}$ transitions disagree with their average value (shown in Table 3) by 3-4 standard deviations each. This may suggest an incorrect placement for one of these two transitions.

Despite the relatively modest data set from the ${ }^{195 \mathrm{~m}} \mathrm{Hg}$ decay, we have been able to make several improvements in the energies and $\beta$ feedings of the levels in ${ }^{195} \mathrm{Au}$ populated by ${ }^{195 \mathrm{~m}} \mathrm{Hg}$; the results of this analysis are listed in Table 4 . In calculating the $\beta$ feedings, we have assumed the NDS value (Zhou, 1999) of $54.2 \pm 2.0 \%$ for the intensity of the ${ }^{195 \mathrm{~m}} \mathrm{Hg}$ isomeric decay branch. This value was deduced from measurement of the relative 
intensity of the $\gamma$ rays in the isomeric and $\beta$ decay branches (see, for example, Vieu, 1973). We have thus normalized the total $\beta$ decay intensity to $45.8 \pm 2.0 \%$.

The decays of the spectroscopy samples were followed over several half-lives to determine the best values of the decay constants for use in obtaining the cross sections. For the ${ }^{195 \mathrm{~g}} \mathrm{Hg}$ decay, 5 different $\gamma$ rays were observed for $24 \mathrm{~h}$. The counting rates for those transitions are shown in Fig. 5. For the ${ }^{195 \mathrm{~m}} \mathrm{Hg}$ decay, 3 different $\gamma$ rays were observed for $5.5 \mathrm{~d}$ as indicated by the results shown in Fig. 6. The individual values of the half-lives and the values of the chi-squared per degree of freedom $\left(\chi^{2} / F\right)$ of the fits are shown in Table 5. The dead-time corrections were checked by normalizing all counting rates to that of the $328.5-\mathrm{keV} \gamma$ ray from the decay of $447-\mathrm{y}{ }^{194} \mathrm{Hg}$. Such corrections amounted to no more than a few tenths of a per cent over the course of the data collecting. As a verification of the dead-time correction, we have determined the half-life of the ${ }^{24} \mathrm{Na}$ that is present in our irradiated samples. Its deduced value is $14.96 \pm$ $0.03 \mathrm{~h}$, in excellent agreement with the presently accepted value of $14.951 \pm 0.003 \mathrm{~h}$.

The currently recommended value for the ${ }^{195 \mathrm{~g}} \mathrm{Hg}$ half-life is $10.53 \pm 0.03 \mathrm{~h}$ based on a measurement reported by Lindenberg et al. (2001). (The published 1999 NDS evaluation shows the value $9.9 \pm 0.5 \mathrm{~h}$, but the online ENSDF evaluation lists the more recent value.) Our average value, with which each of the 5 measured values agrees within one standard deviation, is $10.84 \pm 0.03 \mathrm{~h}$, which is of precision comparable to Lindenberg's value but in substantial disagreement with their value. Our value for the ${ }^{195 \mathrm{~m}} \mathrm{Hg}$ half-life is $41.6 \pm 0.2 \mathrm{~h}$, which agrees with and is considerably more precise than the current NDS 
value of $41.6 \pm 0.8 \mathrm{~h}$. In calculating the $\log f t$ values for the decay of ${ }^{195 g} \mathrm{Hg}$ in Table 3 , we have used the previously accepted half-life of $10.53 \mathrm{~h}$. Using our newly determined half-life would increase the $\log f t$ values by only about 0.01 , which is well within the uncertainties of all of the tabulated values. 


\section{Discussion}

The large thermal cross sections determined in the present work are consistent with values found for other $\mathrm{Hg}$ isotopes as well as for other nuclei in the mass region. It is not uncommon to find thermal cross sections in this region in excess of $100 \mathrm{~b}$, and indeed several even exceed $1000 \mathrm{~b}$, including ${ }^{184} \mathrm{Os}$ and ${ }^{196,199} \mathrm{Hg}$ (Mughabghab, 2006).

In interpreting the measured cross sections, it is instructive to compute the isomer ratios $I R$, defined for thermal and epithermal neutrons in terms of the ground and metastable state cross sections as

$$
I R_{t h}=\frac{\sigma(m)}{\sigma(g)+\sigma(m)}, \quad I R_{e p i}=\frac{I(m)}{I(g)+I(m)}
$$

The presently measured values of the cross sections give

$$
I R_{t h}=0.053 \pm 0.006, I R_{e p i}=0.056 \pm 0.006
$$

where the uncertainty does not include that due to the ${ }^{194} \mathrm{Hg}$ half-life as any variation in that value has the same relative effect on the ground and metastable state cross sections and hence cancels in the isomer ratio.

The agreement of the values of the isomer ratios for thermal and epithermal neutrons is commonly observed in cases in which the cross sections are large enough to be 
dominated by statistical considerations. In this mass region, for example, isomer ratios for thermal and epithermal neutrons for capture by ${ }^{196} \mathrm{Hg}$ give respectively $0.024 \pm 0.002$ and $0.029 \pm 0.003$ (Devlin, Keck, and Krane, to be published) and for capture by ${ }^{196} \mathrm{Pt}$, $0.052 \pm 0.005$ and $0.060 \pm 0.006$ (Petersen, Keck, and Krane, to be published).

Huizenga and Vandenbosch (1960) have proposed a statistical model for the isomer ratios that is based on 3 parameters: the spin of the capture state, the width of the distribution of capture states with that spin, and the number of prompt transitions (assumed to be of dipole character) leading from the capture states to the final observed state. For neutron capture by the spin-0 nuclides ${ }^{194,196} \mathrm{Hg}$ and ${ }^{196} \mathrm{Pt}$, little is known about the resonances but presumably s-wave capture dominates for thermal and epithermal neutrons, so the capture states should all have spin $1 / 2$. Thus the primary contributor to the difference between captures leading to the ground state and the metastable state is due to the spin change that must be accounted for in the cascade of prompt $\gamma$ rays $(\Delta J=0$ and 6 respectively for the ground and metastable states in ${ }^{194,196} \mathrm{Hg}$, and ${ }^{196} \mathrm{Pt}$ ). Under this assumption, the thermal and epithermal isomer ratios in each nuclide should agree, and indeed the measured values do overlap. In each case the slight excess of the isomer ratio for the epithermal neutrons can be understood if these neutrons contribute some p-wave capture leading to $3 / 2$ capture states, thereby reducing the spin change of the cascade to the metastable state by one unit and increasing the value of the corresponding cross section. 


\section{ACKNOWLEDGEMENTS}

The assistance of and support by the Oregon State University Radiation Center in

performing the irradiations is acknowledged with appreciation. A portion of this work was supported by the U.S. Department of Energy under contract DE-FG03-98ER41060. 


\section{References}

Aarnio, P. A., Routti, J. T., Sandberg, J. V., 1988. MicroSAMPO - Personal computer based advanced gamma spectrum analysis system. J. Radioanal. Nucl. Chem. 124, 457465.

Bureau International des Poids et Mesures, 2006. Table of Radionuclides, vols. 1-3.

Farzine, K., v. Buttlar, H., 1974. Levels of ${ }^{195} \mathrm{Au}$ from the decays of ${ }^{195 \mathrm{~g}} \mathrm{Hg}$ and ${ }^{195 \mathrm{~m}} \mathrm{Hg}$. Z. Physik 270, 155-162.

Frána, J., Špalek, A., Fišer, M., Kokeš, A., 1971. Decay of ${ }^{195 m} \mathrm{Hg}$ and ${ }^{195} \mathrm{Hg}$ to levels in ${ }^{195} \mathrm{Au}$. Nucl. Phys. A 165, 625-640.

Helmer, R. G., van der Leun, C., 2000. Recommended standards for $\gamma$-ray energy calibration (1999). Nucl. Instr. Meth. Phys. Res. A 450, 35-70.

Hornshoj, P., Nielsen, H. L., Rud, N., and Ravn, H. L., 1981. The halflife of ${ }^{194} \mathrm{Hg}$ determined by means of quantitative on-line mass separation. Nucl. Inst. Meth. 186, 257259.

Huizenga, J. R., Vandenbosch, R., 1960. Interpretation of isomeric cross-section ratios for $(n, \gamma)$ and $(\gamma, n)$ reactions. Phys. Rev. 120, 1305-1312. 
Lindenberg, K., Neumann, F., Galaviz, D., Mohr, P., Vogt, K., Volz, S., Zilges, A., 2001. Half-lives of $\mathrm{Au}, \mathrm{Hg}$, and $\mathrm{Pb}$ isotopes from photoactivation. Phys. Rev. C 63, 047307-1 047307-4.

Mughabghab, S. F., 2006. Atlas of Neutron Resonances, $5^{\text {th }}$ ed. Elsevier, Amsterdam.

Probst, H.-J., Alderliesten, C., Jahn, P., 1979. A new determination of the half-life of ${ }^{194}$ Hg. Z. Naturforsch. 34a, 387-390.

Vieu, Ch., Peghaire, A., Dionisio, J. S., 1973. Étude de la transmutation ${ }^{195 m} \mathrm{Hg}+{ }^{195} \mathrm{Hg}$ $\rightarrow{ }^{195} \mathrm{Au}$. Revue de Physique Appliquée 8, 231-246.

Zhou, C., Nuclear data sheets for A = 195, 1999. Nuclear Data Sheets 86, 645-784; for a more recent update, see also the on-line compilation of the Evaluated Nuclear Structure Data File: http://www.nndc.bnl.gov/ensdf/. 
Fig. 1. States involved in neutron capture by ${ }^{194} \mathrm{Hg}$ and subsequent decays.

Fig. 2. Low-energy portion of $\gamma$-ray spectrum from ${ }^{195} \mathrm{Hg}$ sample showing prominent peaks from decays of ground (g) and metastable (m) states of ${ }^{195} \mathrm{Hg}$. Other marked peaks are due to the decays of ${ }^{194} \mathrm{Hg}(\mathrm{a}),{ }^{197 \mathrm{~g}} \mathrm{Hg}$ (b), and ${ }^{197 \mathrm{~m}} \mathrm{Hg}$ (c).

Fig. 3. High-energy portion of $\gamma$-ray spectrum from ${ }^{195} \mathrm{Hg}$ sample. Marked peaks are due to decays of ground (g) and metastable (m) states of ${ }^{195} \mathrm{Hg}$ and ${ }^{194} \mathrm{Hg}$ (a).

Fig. 4. Intensities of 401.7- and 869.0-keV $\gamma$ rays in the decay of ${ }^{195 \mathrm{~g}} \mathrm{Hg}$ relative to intensity of 779.8-keV $\gamma$ ray.

Fig. 5. Counting rates vs. time for $5 \gamma$ rays emitted in the decay of ${ }^{195 \mathrm{~g}} \mathrm{Hg}$. Fitted lines are labeled with energies in $\mathrm{keV}$.

Fig. 6. Counting rates vs. time for $3 \gamma$ rays in the decay of ${ }^{195 m} \mathrm{Hg}$. Fitted lines are labeled with energies in keV. The first 4 points have been dropped from the 261.8$\mathrm{keV}$ analysis to eliminate the effect of the component of that line that originates from the decay of ${ }^{195 \mathrm{~g}} \mathrm{Hg}$. 

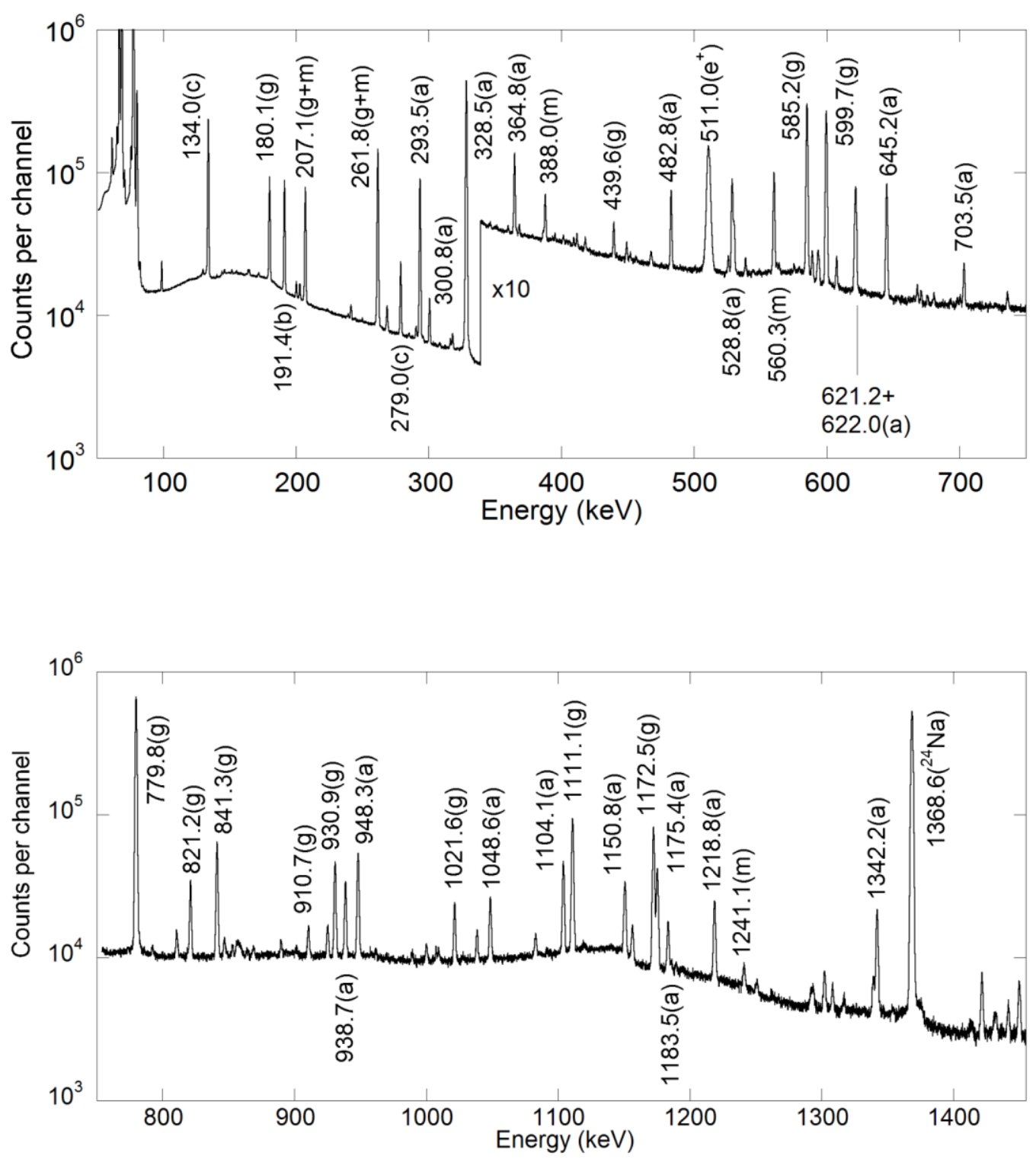


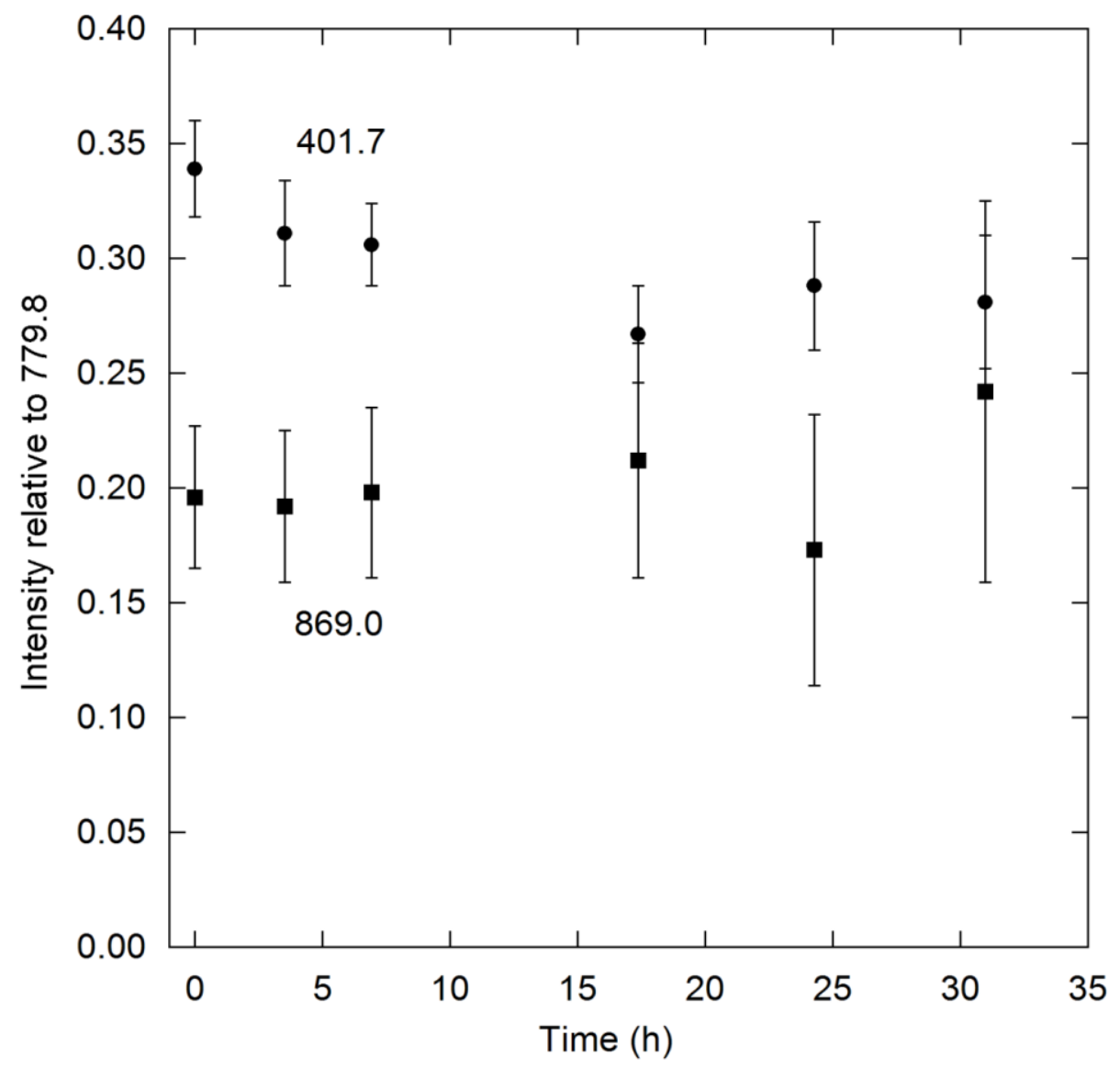




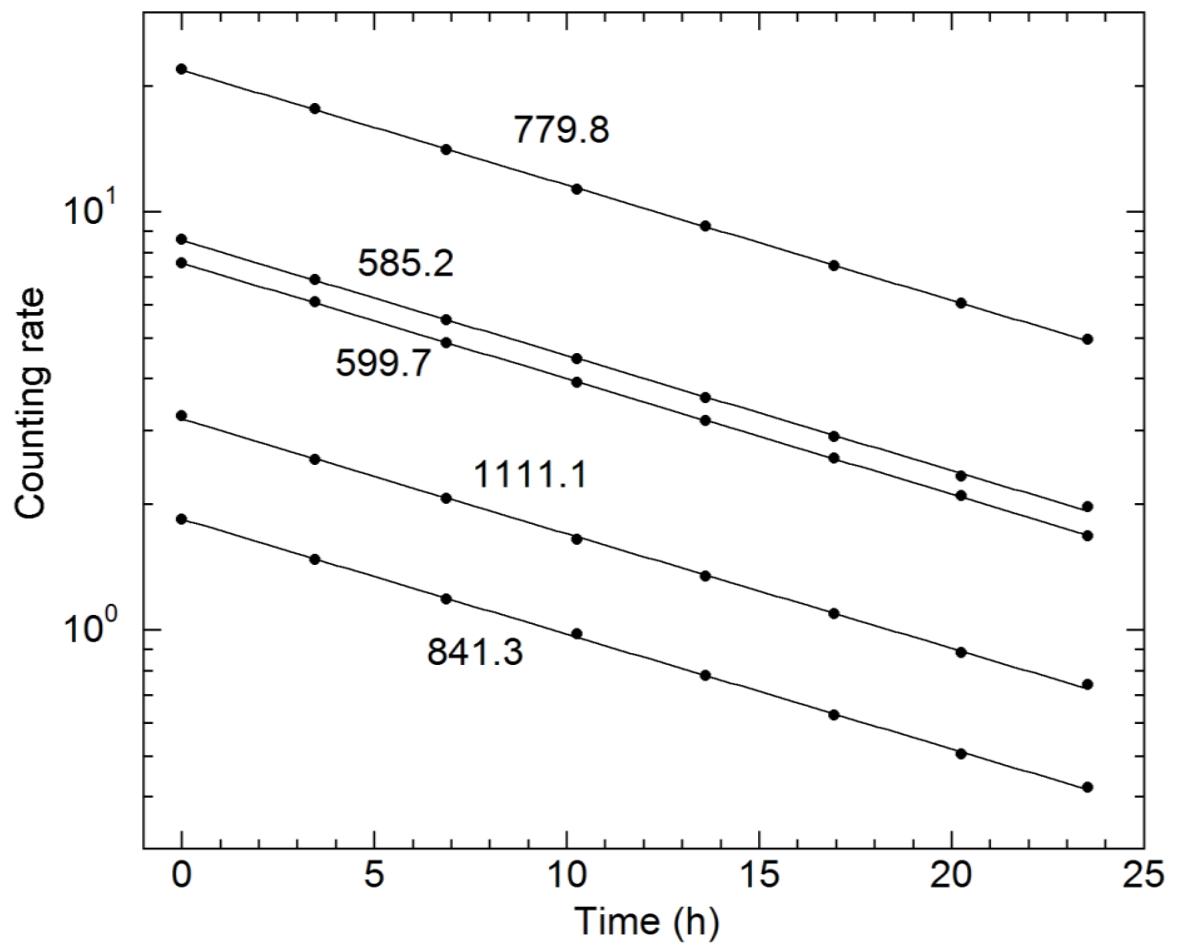




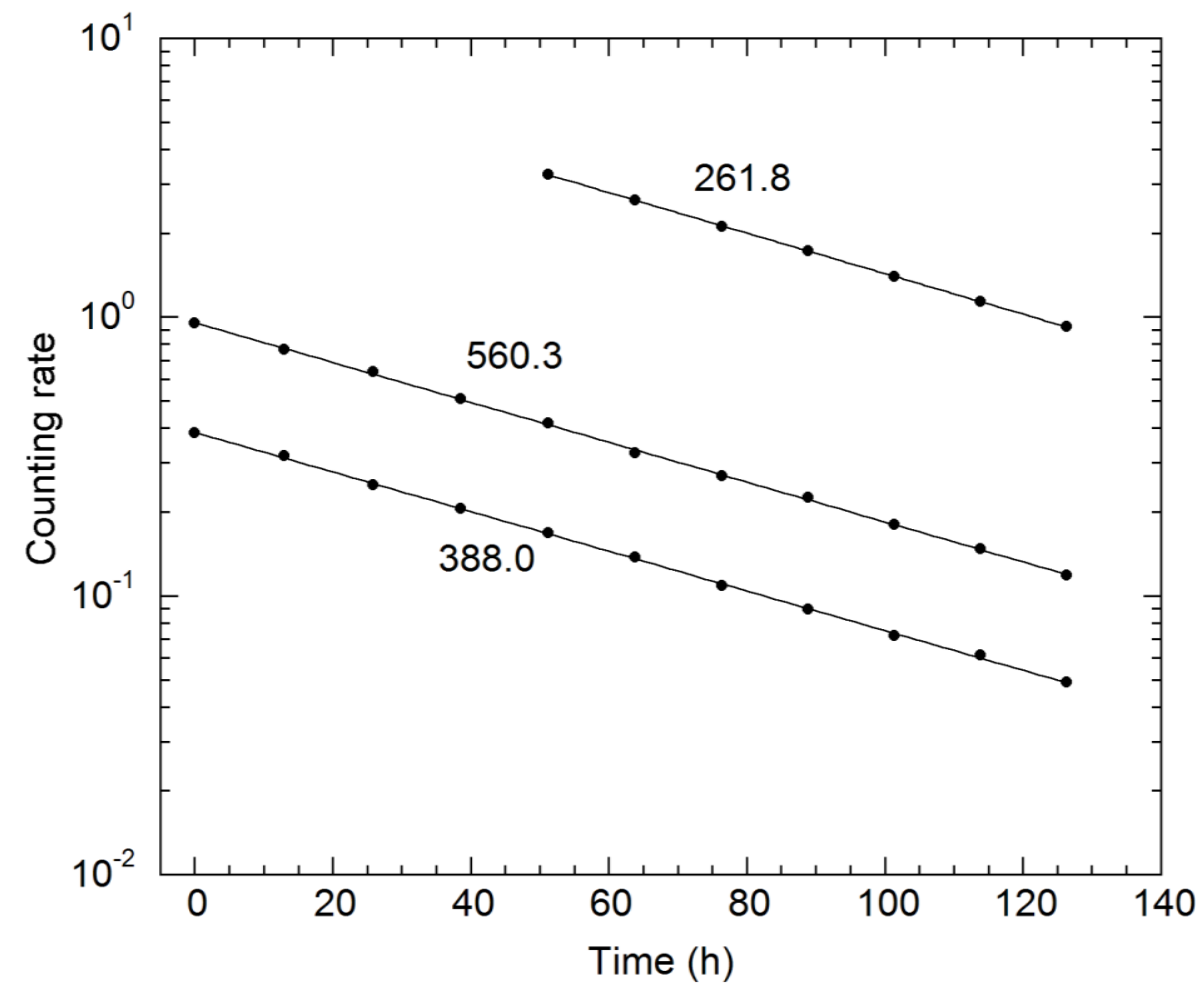




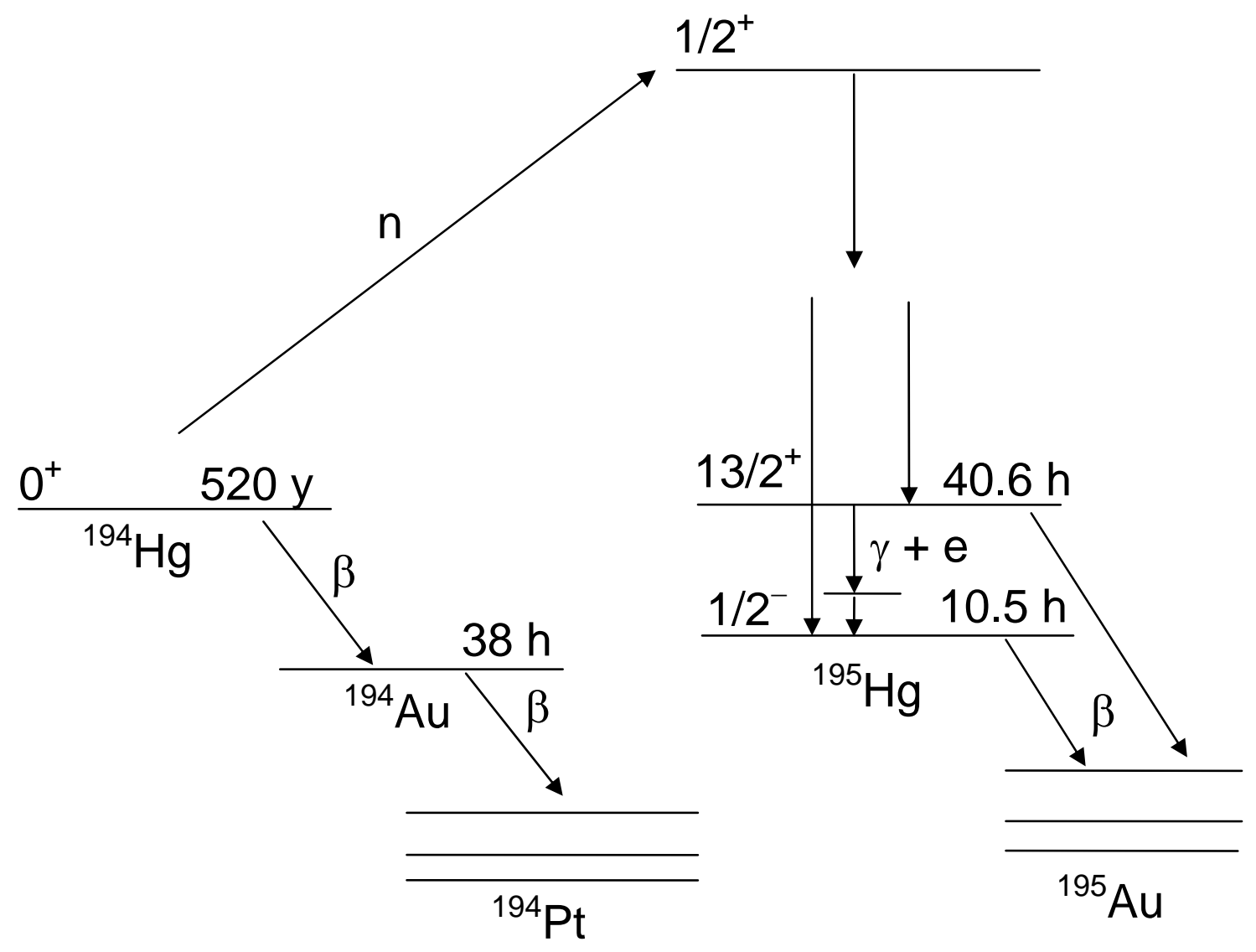

